\title{
WETTABILITY IMPROVEMENT BY PLASMA IRRADIATION AND ITS APPLICATIONS TO PHASE CHANGE PHENOMENA
}

\author{
Y. Takata*, S. Hidaka and M. Kohno \\ *Author for correspondence \\ Department of Mechanical Engineering Science, \\ Faculty of Engineering, Kyushu University \\ 744 Motooka, Nishi-ku, Fukuoka 819-0395, \\ Japan \\ E-mail: takata@mech.kyushu-u.ac.jp
}

\begin{abstract}
Plasma irradiation is one of the techniques to improve surface wettability. This technique can be used to enhance heat transfer of liquid-vapor phase change. For instance, evaporation of water droplet can be enhanced by plasma irradiation. Relation between plasma irradiation time and contact angle was examined first for three metals and then the lifetime of water drop on hot surface was measured changing the surface wettability by plasma irradiation. The lifetime of water drop decreased and the wetting limit temperature increased with the increasing irradiation time of plasma. Hydrophilicity by plasma irradiation is not a permanent effect but it will be useful for enhancement of cooling of hot metal.
\end{abstract}

\section{INTRODUCTION}

In recent years, significant attentions have been paid to the wettability of the surface since it is one of the important parameters affecting phase change heat transfer. Especially, heat transfer community of Japan often organizes special sessions on wettability effects in recent heat transfer conferences.

For exact discussion on the wettability effects, it is needed to extract the effect only of wettability, and however, it is hard to realize it. Conventional methods in experiments were to coat some hydrophilic or hydrophobic materials on the surface to change its contact angle. Even if the coating layer is thin in the order of micron meter, it is impossible to eliminate the effect of thermophysical properties of coating materials in heat transfer measurements.

In 1995, photo-induced superhydrophilicity of $\mathrm{TiO}_{2}$ was discovered and since then, it became possible to change only the surface wettability by exposing its surface to UV light. The authors have been conducting the series of experiments on various phase change heat transfers using this phenomenon[1]. Takamasa et al.[2] discovered another phenomenon called the Radiation Induced Surface Activation (RISA). The RISA effect is that the oxide surface of metals like stainless steel, aluminum and tungsten becomes hydrophilic by radiation of $\gamma$-ray. Their plan is to apply this effect to enhance boiling heat transfer and to increase critical heat flux in nuclear reactor cooling system.

Instead of using UV or $\gamma$-ray, irradiation of plasma can be used to obtain hydrophilic surface instantly. The plasma irradiation is practically applied to the pretreatment of printing, bonding or soldering processes. The authors have proposed an idea of heat transfer enhancement by plasma irradiation and have been conducting a series of experimental studies, e.g. evaporation of water drop on heated surface. The present paper introduces the characteristic of wettability change and applications to phase change heat transfer by plasma irradiation.

\section{CHARACTERISTIC OF WETTABILITY CHANGE BY PLASMA IRRADIATION \\ 2.1 Mechanism of hydrophilicity}

The rinse and cleaning effects by plasma irradiation have been widely studied so far. There are two ways of utilization of plasma for surface reform; one is to reform the surface by making use of the radicals generated by plasma during polymerization reaction, and the other is just to use the radicals without any chemical reactions. The author utilizes the latter process.

Some literatures are available on the effects of plasma irradiation on the surface[3]. Recently, Liu et al.[4] have reported the result of surface element analysis by XPS before and after plasma irradiation to the polymer surface. According to their analysis, hydroxyl, carboxyl and amide radicals that were not observed before irradiation were detected on the surface. These polar radicals increase the surface energy and make the surface hydrophilic. They reported that the contact angle for water and glycol on polyacrylonitrile deceased to $18 \sim 35^{\circ}$ after the irradiation, and however, the terminal angle did not reach to superhydrophilic level. 
In general, a metallic surface has an oxide layer of $30 \sim 40 \AA$ in thickness and a gas absorbed layer of $2 \sim 3 \AA$ on it. Furthermore, there are some organic substances on top as dirt and consequently the surface is hydrophobic due to its low surface energy. By the plasma irradiation, the dirt material on the surface combines with the plasma particle, then it is removed from the surface and finally cleaned surface appears as illustrated in Figure 1(a). Moreover, as shown in Figure 1(b), gas absorbed layer combines with plasma particle and eventually the surface becomes hydrophilic oxide layer. These interactions between plasma particles and surface materials generate microscopic asperities.

However, the hydrophilicity generated by plasma irradiation is not a permanent nature and the surface returns to hydrophobic state as time goes by. It is preferable from the viewpoint of heat transfer enhancement if the surface becomes permanently hydrophilic. Since it is difficult to have a hydrophilic surface for a long period, the applications of this method to the enhancement of heat transfer are fairly limited.

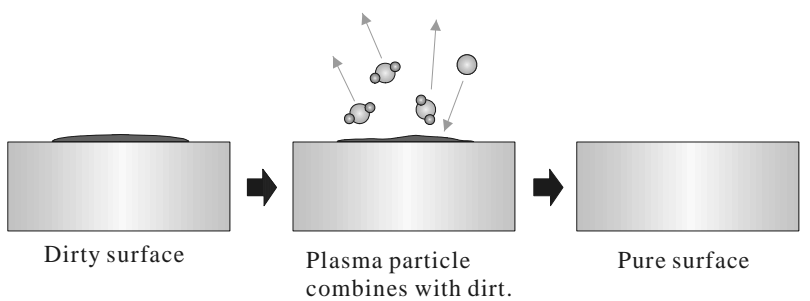

(a) Effect of rinse and cleaning of grease

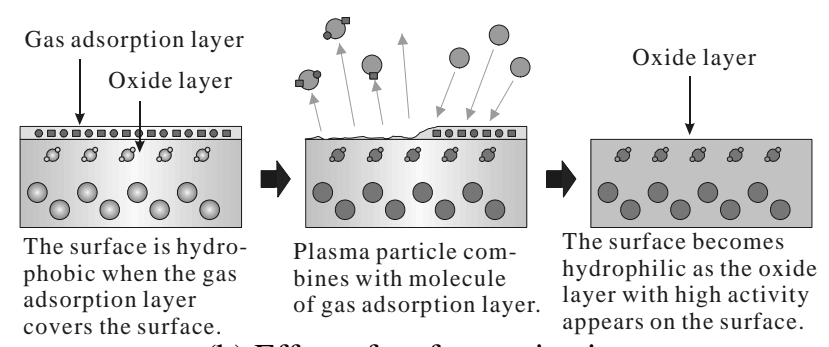

(b) Effect of surface activation

Figure 1: Reaction of the surface by plasma irradiation

\subsection{Plasma generator}

Plasma generator used in the current experiments is a Wettability Reformer ST-700 by Keyence Corp., shown in Figure 2. This reformer is used in pretreatment process of coating or bonding. As shown in Figure 2(b), it has a pair of tungsten electrodes and air nozzle where electric discharge occurs, air is converted to plasma and then blown the target surface. Generated power of the generator is $200 \mathrm{~W}$ and the duration of discharge is adjustable between 10 90 sec. Figure 2(c) shows how to reform the surface by the plasma generator.

\subsection{Surface roughness and change in contact angle}

Improvement of surface wettability by plasma irradiation is very easy and on the contrary, thus improved wettability is not permanent and the surface returns to the original state as time goes by. Characteristics of wettability change depend on materials and in addition, surface roughness changes microscopically by irradiation. Hence, the relationship between surface asperities, change in contact angle and irradiation time should be made clear in advance.

Figure 3 indicates an example of change in surface roughness before and after plasma irradiation. The test materials are copper and aluminum. The test was done with \#600 and mirror finish. It is found form the figure that the surface roughness of aluminum finished with \#600 increases after plasma irradiation and there are no influences for other conditions.

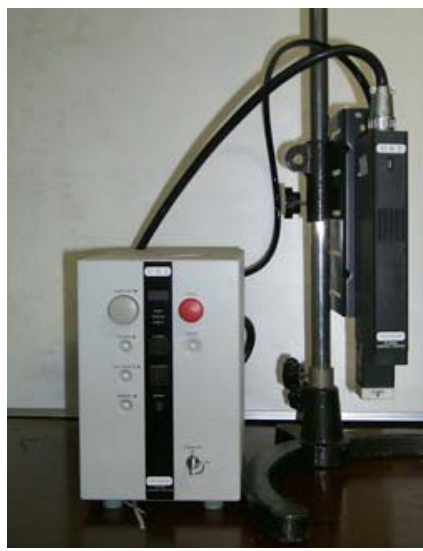

(a) Plasma generator

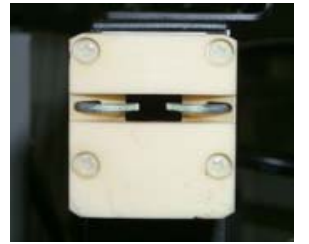

(b) Electrode

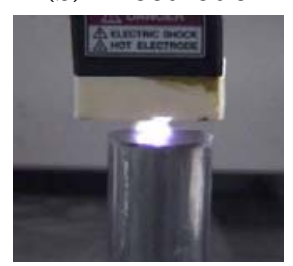

(c) Plasma irradiation
Figure 2: Irradiation to aluminum and the electrode of plasma irradiator

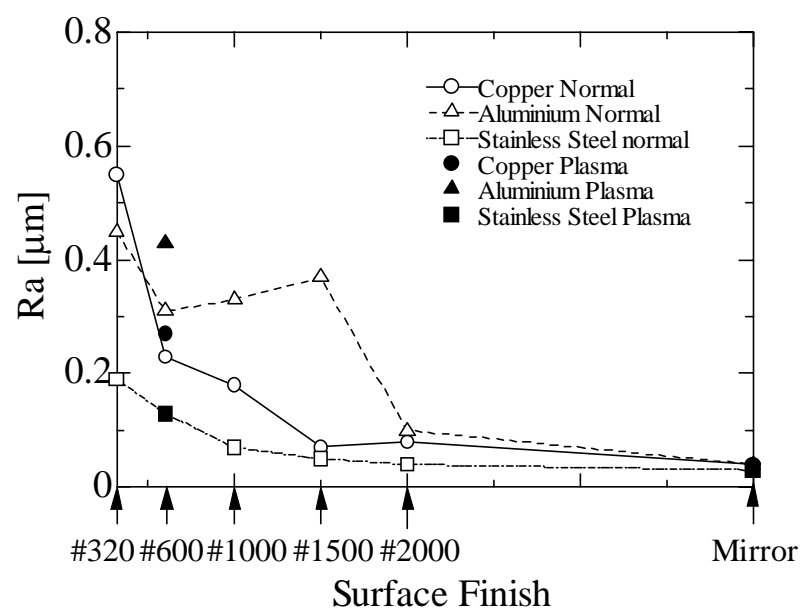

Figure 3: Change in surface roughness

Figure 4 shows characteristics of wettability change for copper and aluminum by plasma irradiation. Horizontal axis is the duration of irradiation and vertical the contact angle for water. Initial contact angles are also indicated in the figure. The contact angle decreases drastically in a few seconds and then gradually with irradiation time. The results obviously depend on materials. For instance, contact angle of copper initially at $102^{\circ}$ decreases to the terminal contact angle of $10^{\circ}$. In addition, the data for copper scatter depending on the difference of 
emery papers. On the other hand, aluminum shows better wettability than copper, and its terminal contact angle is $2 \sim 3^{\circ}$. The result indicates that the aluminum surface finished with \#600 is the best among all data.

As mentioned above, the hydrophilic state by plasma irradiation is not permanent and the surface returns gradually to the original hydrophobic state. Figure 5 shows how to increase the contact angle with elapsed time after terminating the irradiation and it is compared with Figure 4. At a glance, the contact angle of copper considerably scatters and soon returns to higher contact angle. On the other hand, the contact angle of aluminum returns slower compared with copper and its value is kept below $30^{\circ}$ after an hour of termination of irradiation. Generally, the surface finished with \#600 is the most excellent in keeping the contact angle at lower values. Moreover, recovery to hydrophobic state is slower as the irradiation time is longer. Figure 5 shows time up to 5000 sec and it takes more than 2 3days until the surface returns to the original contact angle.

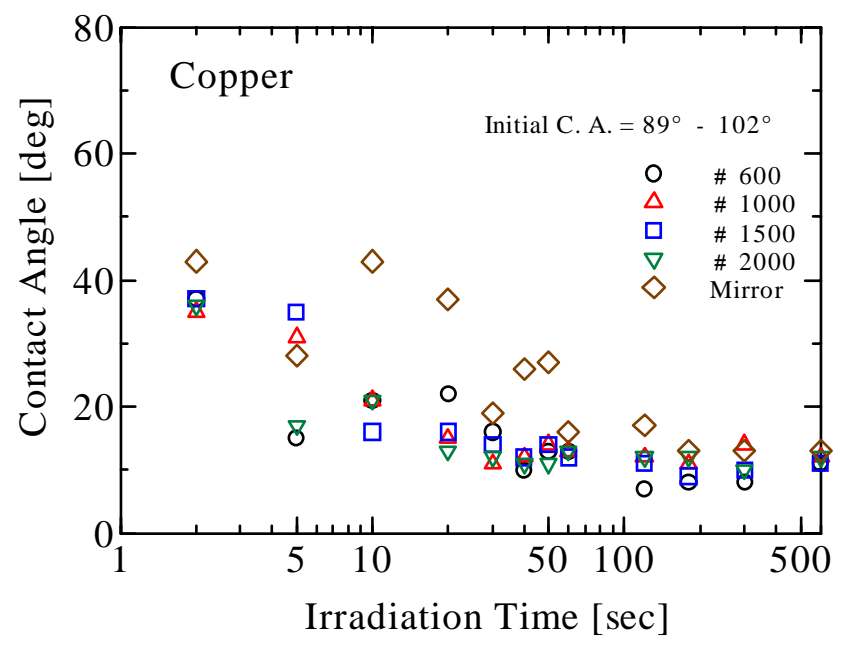

(a) Copper

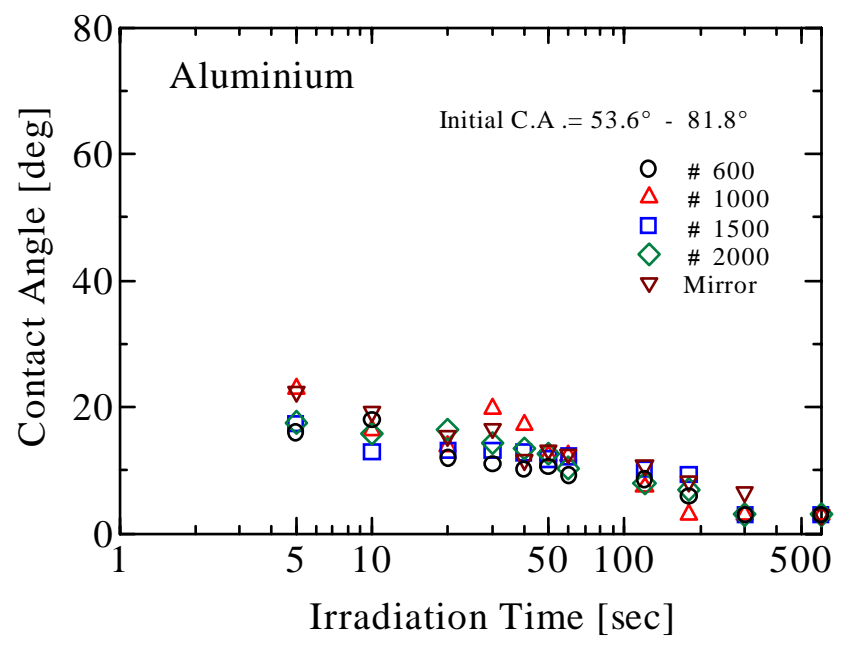

(b) Aluminum

Figure 4: Change in contact angle with irradiation time

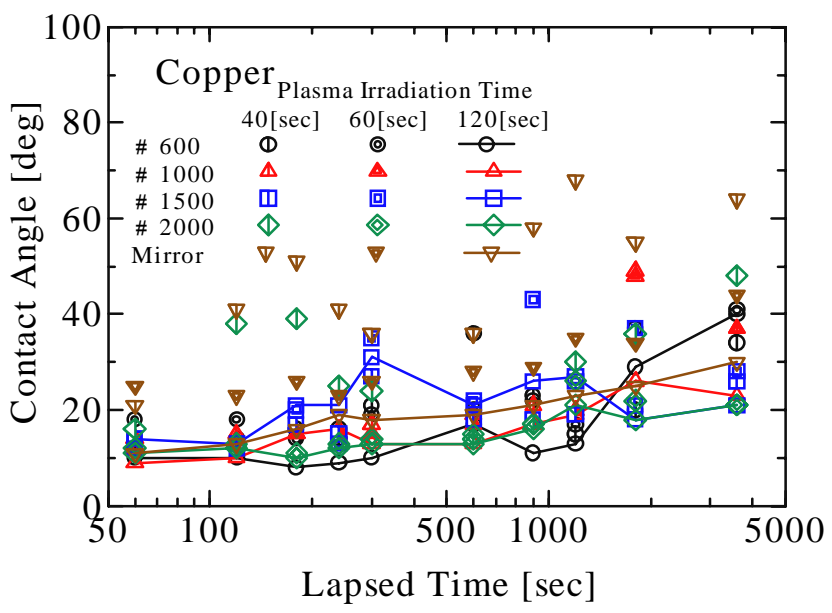

(a) Copper

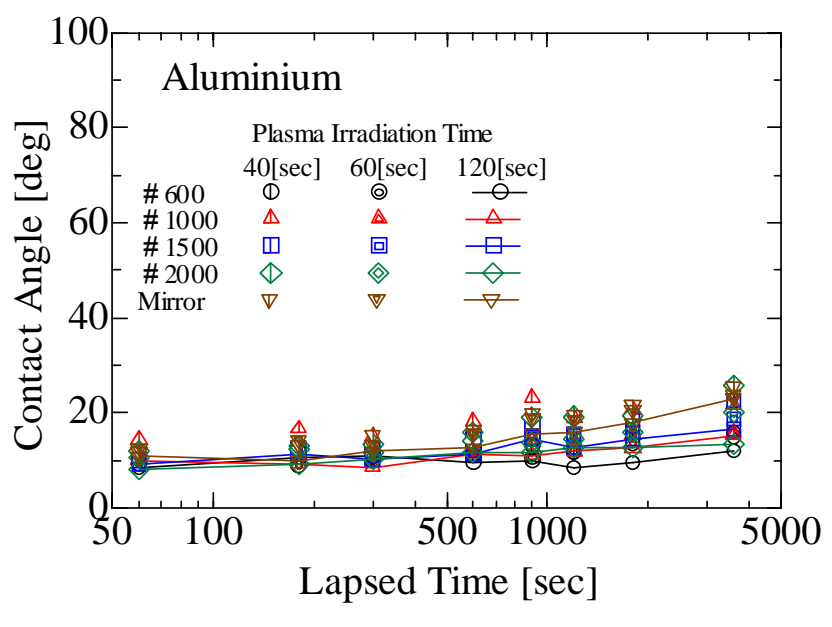

(b) Aluminum

Figure 5: Change in contact angle with lapsed time

\subsection{Aluminum surface changing wettability from superhydrophilic to superhydrophobic state}

As shown in Figure 5, the contact angle of aluminum irradiated by plasma returns to higher angle in relatively short period. From the point of view of heat transfer enhancement, the permanently hydrophilic surface is preferable. We first tried to develop a permanently hydrophilic surface by plasma irradiation. However, the developed surface showed a unique characteristic. It was that the surface changes its wettability from superhydrophilic to superhydrophobic state during about 16 weeks.

This surface is made by the following procedure: First, the bare aluminum surface is polished with emery paper and then irradiated by plasma without removing the grinded powders. Some of these powders are welded on to the surface during irradiation and eventually form the surface with fractal asperity. Figure 6 shows the SEM photographs of the normal and the plasma-reformed surfaces for comparison. The reformed surface has an appropriate roughness that enhances the 
wettability. Figure 7 shows the change in contact angle during 16 weeks. The contact angle increases very gradually with time from $0^{\circ}$ to $150^{\circ}$. Initial state of the surface is superhydrophilic and continues for long hours. It should be noted that the horizontal axis of Figure 7 is "week" and that of Figures 4 and 5 is "sec". If the surface is irradiated by plasma in between, the surface immediately returns to superhydrophilic state and then the angle again increases gradually to superhydrophobic state.

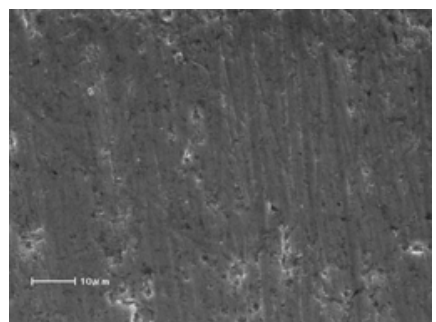

(a) normal

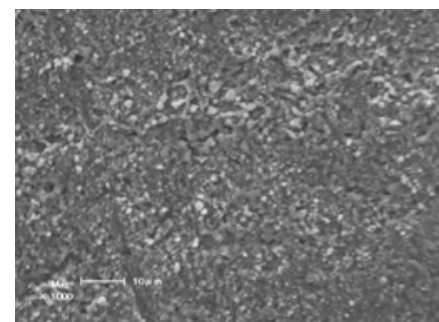

(b) plasma-reformed
Figure 6: SEM picture of aluminum surface

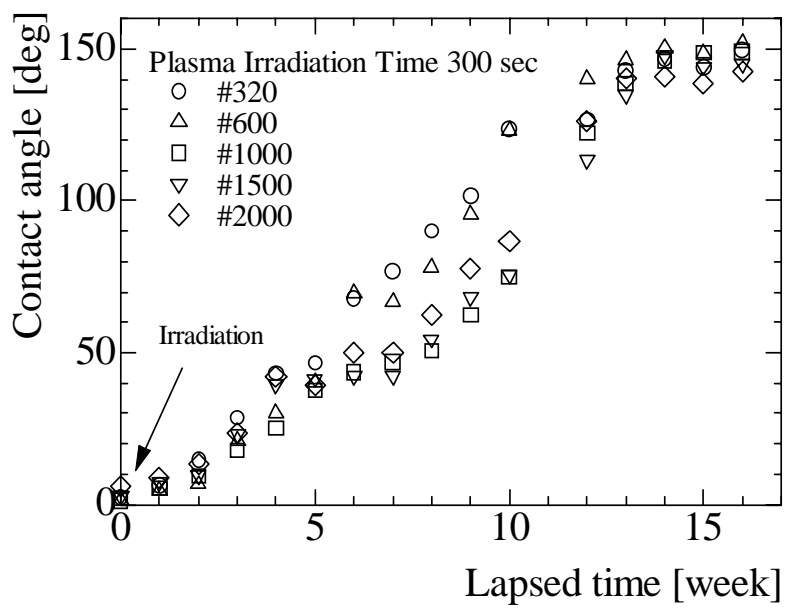

Figure 7: Change in contact angle of plasma-reformed aluminum surface

The contact angle for rough surface, $\theta_{f}$, is given by the Wenzel's equation.

$$
r \sigma_{S}=r \sigma_{L S}+\sigma_{L} \cos \theta_{f}
$$

where $\sigma_{S}$ and $\sigma_{L}$ are the surface energies of solid and liquid, respectively, and $\sigma_{\mathrm{LS}}$ the interfacial energy of liquid and solid. In equation (1), $r$ is called roughness factor and defined as

$$
\cos \theta_{f}=r \cos \theta
$$

where $\theta$ is the contact angle for flat surface. According to equations (1) and (2), $\theta_{f}$ increases for $\theta>90^{\circ}$ and inversely decreases for $\theta<90^{\circ}$.

The reason why this surface shows thus interesting characteristic like Figure 7 has not yet been understood. The author guesses that the surface energy of solid, $\sigma_{S}$, decreases slowly during a long period.

\section{ENHANCEMENT OF PHASE CHANGE HEAT TRANSFER BY PLASMA IRRADIATION}

\subsection{Immersion cooling}

Since the hydrophilicity produced by plasma irradiation is not permanent and the surface returns to its original state as time goes by, the application of this enhancement technique is limited to some extent. Figure 8 illustrates an idea to use it in the cooling process of hot slab in steel-making industries. By installing of plasma generator prior to the cooling zone, the cooling rate can be accelerated by increasing the wettability of the slab surface with plasma irradiation.

To verify this idea, an experiment of immersion cooling was performed using copper sample. Figure 9 is the cooling curve obtained in the experiment. The copper sample is heated up to about $300^{\circ} \mathrm{C}$ in the atmosphere of nitrogen gas and then irradiated by plasma for $120 \mathrm{sec}$ right before immersion. Obviously, the irradiated sample is cooled down more rapidly than the normal sample. Cooling of the irradiated sample finishes in $25 \mathrm{sec}$, while normal one in $34 \mathrm{sec}$. It is confirmed from this simple experiment that the increase in wettability enhances cooling of hot metals.

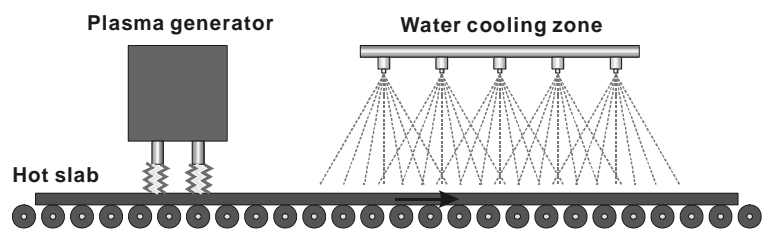

Figure 8: Water cooling of hot slab controlled by plasma irradiation

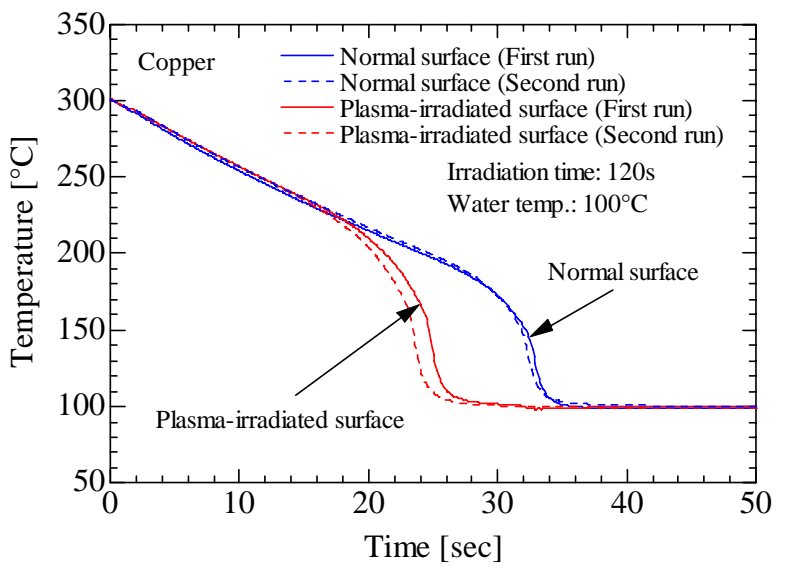

Figure 9: Immersion cooling curve

\subsection{Evaporation of water drop}

Wettability of the solid surface remarkably influences the evaporation rate of water drop[5][6]. On hydrophilic surface the drop spreads widely and forms thin liquid film. In this case, the contact area between solid and liquid increases and consequently the water drop evaporates in a short period. On the contrary, the drop remains spherical shape on the hydrophobic surface and its lifetime becomes longer than that 
of hydrophilic surface. Besides the wettability, thermophysical properties of solid are also important parameters that affect the evaporation rate. The conventional experimental technique was to make use of hydrophobic or hydrophilic coatings to control the surface wettability and eventually, it was impossible to get rid of the effect of thermophysical properties of the coating layer. Thus, it was difficult to examine the effect of wettability only. Since we introduced $\mathrm{TiO}_{2}$ or plasma irradiation to heat transfer experiments, it has been possible to control the surface wettability and extract its effect only.

Figure 10 shows an evaporation curve of water drop on the heated aluminum surface for contact angles of $0 \sim 170^{\circ}$. Drop diameter is $1.64 \mathrm{~mm}$. The contact angle between $0 \sim 90^{\circ}$ is controlled by irradiating plasma to aluminum bare surface and the angle between $110 \sim 170^{\circ}$ is by irradiating to the superwater-repellent-coated aluminum surface. Therefore, a point to notice is that only the data for $0 \sim 90^{\circ}$ are obtained by purely changing contact angle.

As seen from Figure 10, lifetime of water drop decreases as the decreasing contact angle in the region below $120^{\circ} \mathrm{C}$ in temperature, and enhancement of heat transfer by improvement of wettability can be recognized from these data. Referring to the data above wetting limit temperature, the lifetime for $0^{\circ}$ is the smallest among all the contact angles.

Figure 11 indicates the effect of contact angle on wetting limit temperature. The data are for aluminum and copper with three different drop sizes. For both metals, the wetting limit temperature is about $115^{\circ} \mathrm{C}$ for contact angle of $130^{\circ}$, and it increases to $160 \sim 170^{\circ} \mathrm{C}$ for contact angle of $0^{\circ}$. As mentioned above, since the data for $110^{\circ}$ and $130^{\circ}$ were obtained by using super-water-repellent coating on the metal surface, those for $0 \sim 90^{\circ}$ should be compared. The data for $0 \sim 90^{\circ}$ show that the wetting limit temperature increases with the decrease in contact angle.

\section{CONCLUSION}

Plasma irradiation is a handy and attractive tool since it enables to make a solid surface hydrophilic instantaneously. As frequently stated above, however, the effect is temporary and not permanent, and hence, its applications to heat transfer enhancement are limited. Nevertheless, it can be a powerful and strong tool to study the effect of wettability on the wetting limit and Leidenfrost temperatures since it enables to change the wettability only.

The results obtained by the series of experiments are summarized as follows:

1. Surfaces of copper, aluminum and stainless steel become hydrophilic by plasma irradiation. The terminal contact angle differs depending on the materials. The effect continues for a few hours after stopping irradiation.

2. Immersion cooling of hot metal is accelerated by plasma irradiation.

3. The lifetime of water drop on the heated surface decreases significantly by plasma irradiation in the whole temperature range.

4. The wetting limit and the Leidenfrost temperatures increase as the surface wettability increases.

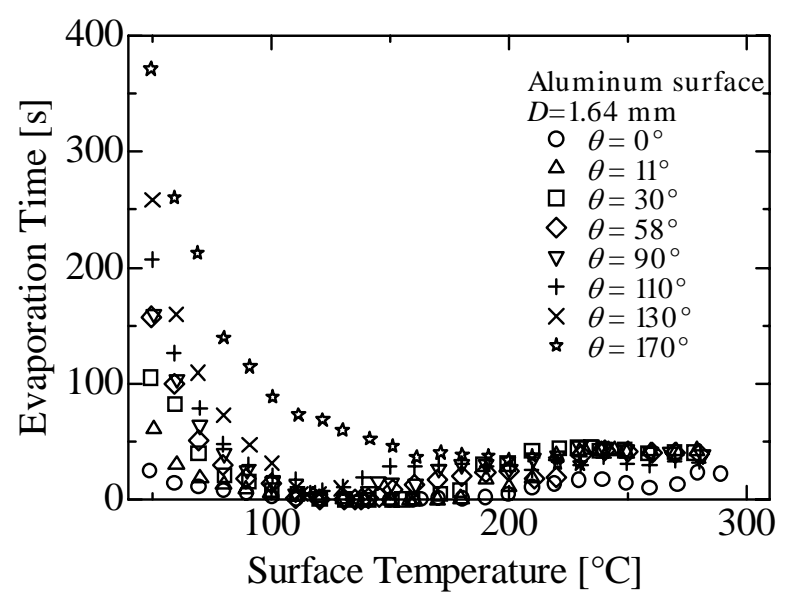

Figure 10: Evaporation curve for various contact angles

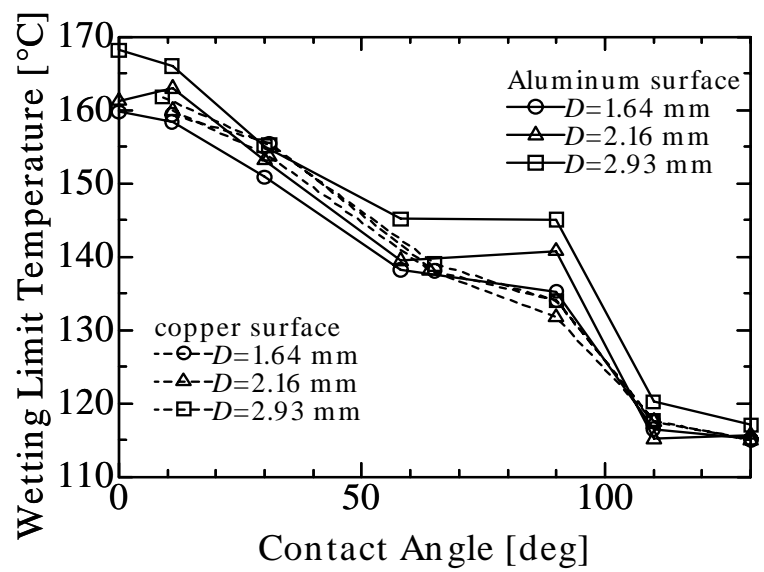

Figure 11: Wettability effect in wetting limit temperature

\section{ACKNOWLEDGEMENTS}

This work was supported partly by the JFE Steel 21st Century Foundation and the Grant-in-Aids for Scientific Research (B) 14350109 and on Priority Area 417 "PhotoFunctional Interface" 14050072 from the Ministry of Science, Sports, Culture and Technology (MEXT).

\section{REFERENCES}

[1] Takata, Y., Surface Wettability Effects in Heat and Fluid Flow, Proceedings of the 4th International Conference on Nanochannels, Microchannels and Minichannels, Paper No. ICNMM2006-96031 (Keynote), 2006

[2] Takamasa, T., Hazuku, T., Okamoto, K., Mishima, K. and Furuya, M., Radiation induced surface activation on Leidenfrost and quenching phenomena, Experimental Thermal and Fluid Science, Vol.29, pp.267-274, 2005

[3] Yamamoto, T., Okubo, M., Imai, N. and Mori, Y., Improvement on Hydrophilic and Hydrophobic Properties of Glass Surface Treated by Nonthermal Plasma Induced by Silent Corona Discharge, Plasma Chemistry and Plasma Processing, Vol.24, No.1, pp.1-12, 2004 
[4] Liu, Y. C. and Lu, D. N., Surface Energy and Wettability of Plasma-treated Polyacrylonitrile Fibers, Plasma Chemistry and Plasma Processing, Vol.26, pp.119-126, 2006

[5] Takata, Y., Hidaka, S., Yamashita, A. and Yamamoto, H., Evaporation of Water Drop on a Plasma-irradiated Hydrophilic Surface, International Journal of Heat and Fluid Flow, Vol.25, No.2, pp.320-328, 2004

[6] Hidaka, S., Yamashita, A. and Takata, Y.,Effect of Contact Angle on Wetting Limit Temperature, Heat Transfer -Asian Research, Vol.35, No.7, pp.513-526, 2006 